Pflegeberufereformgesetz

\section{Bundesrat fordert \\ rasche Reform der Pflegeausbildung}

$\mathrm{D}$

er Bundesrat drängt darauf, dass die Anfang 2016 eingeleitete Reform der Pflegeausbildung nunmehr abgeschlossen wird. In einer Entschließung fordert er Bundestag und Bundesregierung auf, alle notwendigen Anstrengungen zu unternehmen, um das Gesetzgebungsverfahren zum Abschluss zu bringen.

Die Länder begrüßen die Pläne der Bundesregierung für eine überwiegend generalistische Pflegeausbildung, die die drei Ausbildungen in der Altenpflege, der
Gesundheits- und Krankenpflege sowie der Gesundheits- und Kinderkrankenpflege zu einem einheitlichen Berufsbild zusammenfasst. Der Bundesrat hatte hierzu am 26. Februar 2016 Stellung genommen. Derzeit liegt der Gesetzentwurf dem Bundestag zur Beschlussfassung vor. Für die Sicherung einer qualitativen Pflegeversorgung ist eine Reform der Pflegeausbildung dringend erforderlich, betonen die Länder. Darin müssten pflegewissenschaftliche Erkenntnisse und über- greifende Kompetenzen zur Pflege von Menschen aller Altersgruppen in allen Versorgungsbereichen und Pflegesettings vermittelt werden. „Die Entschließung des Bundesrates zeigt, dass auch die Länder hinter dem Vorhaben der Bundesregierung stehen, die drei Pflegeberufe zu einem Berufsbild zusammenzuführen", betont Bundesfamilienministerin Manuela Schwesig. Sie plädiert dafür, das parlamentarische Verfahren ,jetzt schnellstens" abzuschließen. Schwesig verweist auf die „erforderlichen Verbesserungen für die Pflegeausbildung von der Schulgeldfreiheit über die Ausbildungsqualität bis zur Aufwertung des Berufs".

www.bundesrat.de

\title{
INITIATIVE
}

\section{Attraktive Arbeitsbedingungen durch mehr Personal}

Der Deutscher Pflegerat unterstützt die Initiative des Pflegebevollmächtigten der Bundesregierung: Staatssekretär Karl-Josef Laumann ermutigt die Pflegeeinrichtungen, attraktivere Arbeitsbedingungen für die Pflegekräfte zu schaffen.

In einem Schreiben vom Januar 2017 an rund 27.000 ambulante und stationäre Pflegeeinrichtungen zeigt der Pflegebevollmächtigte Chancen auf, wie die Einrichtungen die aktuellen Pflegereformen nutzen können, um die Rahmenbedingungen für die professionell Pflegenden zu verbessern. Dazu gehören für Laumann vor allem flächendeckend faire Löhne, weniger unfreiwillige Teilzeitbeschäftigungen und die Entbürokratisierung der Pflegedokumentation. Bei der Umsetzung dieser Ziele sei man „natürlich auf die Unterstützung der Einrichtungsbetreiber und der Pflegekräfte angewiesen", wofür Laumann diesen seinen Dank ausspricht.

Bessere Bezahlung: Laumann weist in dem Schreiben u.a. darauf hin, dass mit den Pflegestärkungsgesetzen ein wichtiger Grundstein für eine bessere Bezahlung der Altenpflegekräfte geschaffen worden sei. Bereits seit 2015 gelte: Pflegeeinrichtungen, die nach Tarif bezahlen, bekommen die Gehälter von den Pflegekassen und den Sozialhilfeträgern in der Pflegevergütung finanziert. Seit 1. Januar 2017 gelte diese Regelung bei der Bezahlung von Gehältern bis zur Höhe von Tariflöhnen auch für nicht-tarifgebundene Einrichtungen.
Unfreiwillige Teilzeit abbauen: Um dem Problem unfreiwilliger Teilzeit zu begegnen, weist der Staatssekretär im Bundesgesundheitsministerium auf den Abschluss von Gesamtversorgungsverträgen hin. "Gerade in den ostdeutschen Bundesländern arbeiten viele Pflegekräfte nur deshalb in Teilzeit, weil sie keine Vollzeitstelle finden. Mit dem Dritten Pflegestärkungsgesetz haben wir deshalb klargestellt, dass Einrichtungs- und Kostenträger auch Gesamtversorgungsverträge abschließen können. Dadurch können Mitarbeiter eines Trägers flexibler in mehreren Versorgungsbereichen eingesetzt werden - etwa auch in der Tages- und Kurzzeitpflege", erklärt Laumann.

Mehr Kolleginnen und Kollegen gefordert: Der DPR unterstützt die Initiative des Pflegebevollmächtigten der Bundesregierung für attraktivere Arbeitsbedingungen des Pflegepersonals. Entscheidend für die Verbesserung der Rahmenbedingungen für die professionell Pflegenden ist es jedoch auch, dass „,Mehr Kolleginnen und Kollegen" da sind. Eine Verbesserung der Personalausstattung in den Pflegeeinrichtungen ist daher dringend geboten. Der neue Pflegebedürftigkeitsbegriff sorgt für höhere Leistungsan- sprüche der Pflegebedürftigen. Leistbar sind diese nur mit deutlich mehr Personal. Als DPR hätten wir uns gewünscht, dass der Gesetzgeber in den letzten Jahren auf die Personalfrage ein stärkeres Augenmerk gelegt hätte. Das für das Jahr 2020 geplante, recht unverbindliche Personalbemessungssystem kommt eindeutig zu spät. Die stationären Pflegeeinrichtungen benötigen schnellstmöglich einen einheitlichen Personalschlüssel, der auch finanziell abgesichert ist.

Forschungsvorhaben nötig: Die Schaffung bestmöglicher Arbeitsbedingungen in der Pflege ist eine entscheidende $\mathrm{Zu}$ kunftsaufgabe. Der DPR fordert daher ein auf Bundesebene angesiedeltes, umfassendes Forschungsvorhaben zur zentralen Frage „Wie sieht angesichts knapper Personalkapazitäten die Arbeitsgestaltung und Arbeitsorganisation der Pflege in der Zukunft aus?". Diese Frage gilt es, angesiedelt beim Pflegebevollmächtigten, schnell und umfassend zu lösen. Das hervorragend funktionierende Projekt zur Entbürokratisierung der Pflegedokumentation kann hier Vorbild sein.

www.bmg.bund.de 